\title{
Effect of Modified Atmosphere Packaging on Aril Physico-chemical and Microbial Properties of Two Pomegranate Cultivars (Punica granatum L.) Grown in Iran
}

\author{
Sedighe Tavasoli Talarposhti ${ }^{1}$, Mohsen Barzegar ${ }^{1}{ }^{*}$, Zohreh Hamidi-Esfahani $^{1}$ \\ 1- Faculty of Agriculture, Tarbiat Modares University, Tehran, Iran.
}

\section{A B S T R A C T}

Background and Objectives: Edible parts of pomegranate fruits are a rich source of bioactive compounds. The present research examines the effect of modified atmosphere packaging on the fruit physico-chemical and microbial properties of two commercial pomegranate cultivars grown in Iran.

Materials and Methods: The arils were packaged and stored under four different atmospheres. All of the packaged samples were stored at $4{ }^{\circ} \mathrm{C}$ for 15 days.

Results: The results revealed an increase in total acidity of all treatments. The highest total soluble solid (TSS) was observed in 'Yousef-Khani' stored in $10 \% \mathrm{O}_{2}+15 \% \mathrm{CO}_{2}$, while 'Malas-e-Saveh' treated with $20 \% \mathrm{O}_{2}+$ $5 \% \mathrm{CO}_{2}$ showed the highest degree of TSS. The mean value of a* in 'Malas-e-Saveh' arils packed with normal and $\left(15 \% \mathrm{O}_{2}+10 \% \mathrm{CO}_{2}+75 \% \mathrm{~N}_{2}\right)$ atmosphere increased significantly. The $\mathrm{L}^{*}$ showed a decrease in 'YousefKhani'. Total phenolic compounds gradually increased during storage. After storage, decreases in total anthocyanin contents ranged from 12 to $30 \%$ for 'Yousef-Khani'. The overall antioxidant activity of arils in 'Yousef-Khani' showed a 6-15\% increase during storage. However, a reverse effect was observed for 'Malas-eSaveh'. The lowest microbial counts were recorded under the atmosphere containing 10 and $15 \% \mathrm{CO}_{2}$.

Conclusions: Packaging of 'Malas-e-Saveh' arils in 15\% $\mathrm{O}_{2}+10 \% \mathrm{CO}_{2}$ and 'Yousef-Khani' in $15 \% \mathrm{O}_{2}+10 \%$ $\mathrm{CO}_{2}$ or $10 \% \mathrm{O}_{2}+15 \% \mathrm{CO}_{2}$ is recommended to extend the shelf-life of ready-to-eat arils.

Keywords: Pomegranate, Modified atmosphere, Phenolic compounds, Anthocyanin, Antioxidant activity

\section{Introduction}

The pomegranate (Punica granatum L.), cultivated extensively in tropical and subtropical countries, is widely recognized as one of the oldest fruit crops known to humans. There is a broad consensus that the pomegranate fruit is native to Iran, and from there, it has diversified into many other countries, including China, India, Greece, and Spain $(1,2)$. Based on statistical data, the total pomegranate production of Iran in 2013 was $~ 910,000$ tons (3). During the past decade, there has been a considerable increase in the commercial production of pomegranates worldwide, which could be mainly attributed to its unique sensory properties and potential health benefits. The edible parts of pomegranate fruit are a rich source of acids, sugars, vitamins, polysaccharides, polyphenols, essential minerals, and various kinds of antioxidants, including anthocyanins and ellagitannins, ellagic acid, punicalagin and punicalin. These compounds have the potential to inhibit oxidative stress, have a positive effect on controlling the risk factors for cardiovascular diseases (CVDs), and can stop the progress of such diseases $(4,5)$.

Despite the above-mentioned beneficial health effects, there are some reasons, which have deterred the widespread consumption of pomegranates. These include the difficulty of extracting the arils (6), and high concentrations of phenolic compounds in the husk (7), as well as oxidative enzymes that could stain and sometimes irritate sensitive skin, when removing the outer covering of the fruit. It seems that industrial processing of pomegranates could be regarded as a highly productive method to obtain ready-to-eat arils, with intact sensory properties and nutritive values. This, in turn, can increase the production and consumption of pomegranates. Moreover, packaging of pomegranate arils makes it possible to exclude 
those fruits with external defects (bruised, cracked, and sunburnt) that are considered commercially unacceptable and unsuitable for fresh marketing. Accordingly, to meet the increasing demand from consumers for fresh-cut and ready-to-eat fruits (8), minimal processing of pomegranates has gained popularity among both producers and those who buy and use these products. Modified atmosphere packaging (MAP), defined as a packaging technology that modifies or alters the gas composition around the products in food packages, has occupied an important place (9). The principal objective of MAP is to produce a product with minimal processing and fresh characteristics similar to those at harvest, especially flavor, color and texture. It also aims to extend the post-harvest shelf-life of the fruit and reduce the microbial hazards yet maintaining the sensory and nutritional properties.

Many studies have been conducted to examine the potential effect of MAP on both quality preservation and shelf-life extension in fruits (10-13). Gil et al. (1996) reported a $12.92 \%$ decrease in the total anthocyanin content of pomegranate arils after 7 days of storage at $4^{\circ} \mathrm{C}$ and under an atmospheric composition of $13.5 \% \quad \mathrm{O}_{2}$ plus $7.5 \% \quad \mathrm{CO}_{2}$ using polypropylene (PP) baskets (7). López-Rubira et al. (2005) found a $2.46 \%$ increase in total anthocyanin content of pomegranate arils harvested in October, UV-C treated and stored under MAP conditions (26.9-29.2kPa $\mathrm{CO}_{2}$ plus $<1 \mathrm{kPa} \mathrm{O}$ ) after 15 days of storage (6). The authors estimated a minimum of 10 days shelf-life for pomegranate arils stored under MAP at $1^{\circ} \mathrm{C}$. Garcia et al. (2000) suggested that MAP of pomegranate arils at $4^{\circ} \mathrm{C}$ and under the $7 \% \mathrm{O}_{2}$ plus $15 \% \mathrm{CO}_{2}$ with semi-permeable polymer cannot significantly prolong the shelf-life of minimally processed arils (14). Moreover, after 10 days of storage at $5{ }^{\circ} \mathrm{C}$ with modified atmosphere $\left(6.5 \% \mathrm{O}_{2}+\right.$ $11.4 \% \mathrm{CO}_{2}$ ) in polypropylene, no significant change, except for an increase in acidity, was found in the chemical activities of pomegranate arils. Despite the studies mentioned above, there is still particular concern about the use and influence of atmosphere modification on the functional and bioactive properties (e.g. anthocyanins) of fresh-cut products, in general, and pomegranate arils, in particular.

In spite of Iran's enormous success as an exporter and producer of pomegranates in the world, there is not enough information about the potential effect of MAP on the physico-chemical properties of Iranian cultivars.
Therefore, the present study aims to investigate the effect of MAP and storage on the physico-chemical and microbial properties of pomegranate arils of two Iranian commercial cultivars (Malas-e-Saveh and Yousef-Khani).

\section{Materials and Methods}

Chemicals: The anthocyanins standards were purchased from Apin Chemicals Co. Ltd., (Oxfordshire, England), and HPLC grade Methanol $(\mathrm{MeOH})$ was purchased from Caledon Laboratories (Ontario, Canada). Formic acid, acetonitrile, sodium hydroxide, Folin-Ciocalteu reagent, and plate count agar (PCA) were obtained from Merck Chemical Co. (Darmstadt, Germany). Radical 2, 2-diphenyl-1picrylhydrazyl (DPPH) was purchased from Fluka (Germany).

Sample preparation: Two commercial pomegranate cultivars ('Malas-e-Saveh' and 'Yousef-Khani') were purchased from the Agricultural Research Centre of Saveh, Iran. The fruits were transported, on the same day, by a ventilated car to a laboratory at Tarbiat Modares University (Tehran, Iran). After discarding defective ones visually, each fruit was thoroughly rinsed with cold water and drained; then the arils were manually extracted. The separated and homogenized arils were vacuum-packaged, using a packaging machine (Henkelman model 200, USA), under four different atmospheres: (A) $\left(20 \% \mathrm{O}_{2}+5 \% \mathrm{CO}_{2}+75 \%\right.$ $\left.\mathrm{N}_{2}\right)$, (B) $\left(15 \% \mathrm{O}_{2}+10 \% \mathrm{CO}_{2}+75 \% \mathrm{~N}_{2}\right)$, (C) $(10 \%$ $\mathrm{O}_{2}+15 \% \mathrm{CO}_{2}+75 \% \mathrm{~N}_{2}$ ), and normal atmosphere. Moreover, we used a 3-layer coating comprising polyethylene (LDPE), polyamide, and polyethylene (thickness $60 \mu \mathrm{m}$ ) (Tahavol Kala Novin Co. (Nadipack, Iran).

The vacuum-sealing machine utilized (MAP) in the present study relies on a technology that automatically carries out the vacuum process by extracting the air from the product once the lid is closed. Then the cycle completes by adding a gas immediately followed by sealing of the package. A mixture of nitrogen $\left(\mathrm{N}_{2}\right)$, carbon dioxide $\left(\mathrm{CO}_{2}\right)$ and oxygen $\left(\mathrm{O}_{2}\right)$ is then added using a pump. All the packed samples were stored at $4{ }^{\circ} \mathrm{C}$ for 15 days. Microbial and chemical properties of the samples were determined before and after packaging on days 5,10 and 15 of storage. In each stage, the samples' juice was centrifuged $(10000 \mathrm{rpm})$ for $2 \mathrm{~min}$ at $-4{ }^{\circ} \mathrm{C}$ (4).

Determination of physico-chemical properties: The amount of titratable acidity was determined potentiometrically using $0.1 \mathrm{M} \mathrm{NaOH}$ to the titration 
end point of $\mathrm{pH} 8.1$ and was expressed as \%citric acid (4). A refractometer was employed to measure total soluble solids (TSS), expressed as ${ }^{\circ}$ Brix, at $20^{\circ} \mathrm{C}$.

Color: Based on the method described by Alighourchi and Barzegar (2009), aril color was evaluated using a colorimeter (Colourflex, VA, USA). Furthermore, CIELAB parameters, L* (lightness and darkness), $\mathrm{a}^{*}$ (redness and greenness) and $\mathrm{b}^{*}$ (yellowness and blueness) were reported (15).

Total phenolic content: Total polyphenols were estimated colorimetrically according to FolinCiocalteu method, as described in Mousavinejad et al. (2009), using gallic acid as a standard. The results were expressed as mg gallic acid $\mathrm{L}^{-1}$ juice. Moreover, absorption was measured at $760 \mathrm{~nm}$ (1).

Individual and total anthocyanins: The samples anthocyanins were separated and determined by HPLC equipped with Empower Software (Waters Corp., Milford, USA), UV-Vis detector and a Waters $600 \mathrm{E}$ high-pressure pump. The separation was done by a Nucleodur C18 Gravity column $(250 \mathrm{~mm} \times 4.6$ $\mathrm{mm}$, dp $5 \mu \mathrm{m}$ ) from Macherey-Nagel (Düren, Germany). Prior to HPLC injection, the juice samples were centrifuged $(10000 \mathrm{rpm})$ for $2 \mathrm{~min}$ at $4^{\circ} \mathrm{C}$ and passed through a Sep-pak C18 cartridge (Millipore, Milford, USA). A Millipore Swinnex type filter (pore size $=0.45 \mu \mathrm{m})$ was used to remove the particles. After that, $20 \mu \mathrm{L}$ of clarified juice was injected onto the HPLC with three replicates. The separations were performed using gradient HPLC method with the following mobile phase program: formic acid 10\% (A) and acetonitrile (B). The linear gradient started from $95 \% \mathrm{~A}$ and $5 \% \mathrm{~B}$ for $1.67 \mathrm{~min}$; $90 \% \mathrm{~A}$ and $10 \%$ B for $3.34 \mathrm{~min}$; $80 \% \mathrm{~A}$ and $20 \% \mathrm{~B}$ for $20 \mathrm{~min}$; and finally, 95\% $\mathrm{A}$ and $5 \% \mathrm{~B}$ for $25 \mathrm{~min}$. The flow rate was $0.8 \mathrm{~mL} \mathrm{~min}^{-1}$, and the chromatograms were recorded at $520 \mathrm{~nm}$. It is to be pointed out that the methodology reported by Del Carpio Jimenez et al. (2011), which was further improved by Alighourchi $e t$ al. (2013), was applied to determine the anthocyanins $(16,17)$. Anthocyanins were identified by comparing their retention times with those of pure standards, and the their concentration was calculated using an external standard method. Finally, total anthocyanins were estimated by adding the amounts of the six individual anthocyanins previously identified in each chromatogram.

Total antioxidant activity: The antioxidant activity was assessed by DPPH radical scavenging method (18). The absorbance values of the final solutions were determined at $517 \mathrm{~nm}$ by a UV-Visible spectrophotometer (SCINCO, Seoul, South Korea). Moreover, radical scavenging activity was presented as inhibition percentage. The antioxidant activity was calculated using the following equation:

Antioxidant activity $(\%)=\left[1-\left(\mathrm{A}_{\text {sample }} / \mathrm{A}_{\text {control }}\right)\right] \times 100$

Microbial analysis: Microbiological quality was assessed by total plate count. To enumerate the microbial load present in the arils, the samples were serially diluted and plated by pour-plating on plate count agar (PCA, Merck). Agar plates were incubated at $30^{\circ} \mathrm{C}$ for $48-72 \mathrm{~h}$. The results are presented as $\log$ $\mathrm{CFU} \mathrm{mL} \mathrm{m}^{-1}$ (6).

Statistical analysis: A minimum of three replicates were used for each treatment. The experimental data obtained were analyzed by the Statistical Analysis System (SAS) software using analysis of variance (ANOVA) and the differences among means were determined for significance at $P<0.01$. The LSD Multiple Range Test was also applied when appropriate.

\section{Results}

Total soluble solids (TSS) and total acidity (TA): At harvest, TA values for fresh-cut Malas-e-Saveh and Yousef-Khani cultivars were $1.05 \pm 0.00$ and $0.71 \pm 0.01$ (\% citric acid), respectively. As indicated in Figure 1, there was a significant increase $(P<0.01)$ in the TA of both Malas-e-Saveh and Yousef-Khani cultivars at the end of the storage time. These results agree with those of Gil et al. (1996) and Ding et al. (2013) in which Dabai fruit (Canarium odontophyllum Miq.) samples were stored under modified atmosphere conditions $(7,11)$.

The initial TSS $\left({ }^{\circ}\right.$ Brix) values of fresh-cut 'Malase-Saveh' and 'Yousef-Khani' arils were 17.00 \pm 0.10 and $17.15 \pm 0.05$, respectively. After 15 days of storage, the highest level of TSS was observed in cv. Yousef-Khani stored in $10 \% \quad \mathrm{O}_{2}$ plus $15 \% \quad \mathrm{CO}_{2}$. However, the differences among the three treatments (A and $\mathrm{C}$; $\mathrm{A}$ and $\mathrm{B}$; and control) as compared to fresh-cut fruit were not statistically significant. At the end of storage time, no significant difference was observed in the TSS values of Malas-e-Saveh in comparison with the fresh-cut fruit (treated with $20 \%$ $\mathrm{O}_{2}$ plus $5 \% \mathrm{CO}_{2}$. However, decreases in TSS values were observed for the other treatments. 

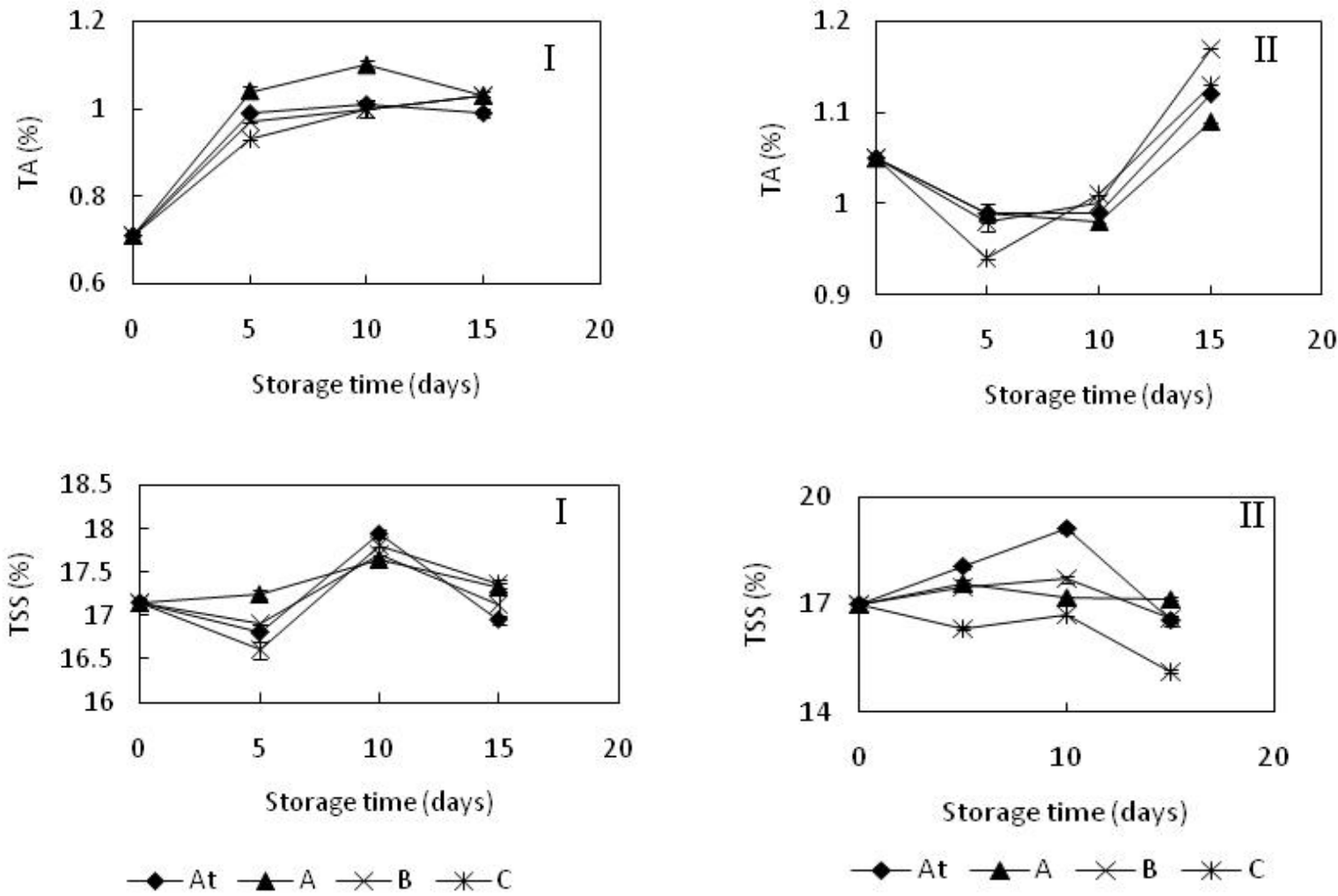

Figure 1. Evaluation of titrable acidity (TA) and total soluble solids (TSS) of pomegranate arils packed under modified atmosphere and control during storage. I: cv. Yousef-Khani, II: cv. Malas-e-Saveh; At: normal air; A: 20:5:75 $\mathrm{O}_{2}: \mathrm{CO}_{2}: \mathrm{N}_{2} ; \mathrm{B}: 15: 10: 75 \mathrm{O}_{2}: \mathrm{CO}_{2}: \mathrm{N}_{2}$; and $\mathrm{C}: 10: 15: 75 \mathrm{O}_{2}: \mathrm{CO}_{2}: \mathrm{N}_{2}$.

Changes in pomegranate color: The values for CIE $\mathrm{a}^{*}$ (redness), $\mathrm{b}^{*}$ (yellowness) and $\mathrm{L}^{*}$ (lightness) parameters for pomegranate arils both before and after packaging are presented in Figure 2. According to the color evaluations, a* of 'Yousef-Khani' arils decreased in all atmospheres while air-stored 'Malase-Saveh' arils and those treated with $15 \% \quad \mathrm{O}_{2}$ plus $10 \% \mathrm{CO}_{2}$ showed higher red color at the end of the storage time. There were no significant differences in $b^{*}$ value of 'Yousef-Khani' arils among different treatments. However, 'Malas-e-Saveh' arils showed significantly lower yellowness. The $\mathrm{L}^{*}$ value of 'Yousef-Khani' arils was reduced while higher levels of L* were observed for 'Malas-e-Saveh' arils (Figure 2).

Evaluation of total phenolic content: The initial total phenolic content values varied ranging from $1040.00 \pm 30.00$ to $953.30 \pm 42.90\left(\mathrm{mg}\right.$ gallic acid $\mathrm{L}^{-1}$ juice) for the arils of 'Yousef-Khani' and 'Malas-eSaveh', respectively. As seen from Figure 3, at the $5^{\text {th }}$ day of storage, the TPC of pomegranate arils decreased significantly as compared to the initial levels however; it then started to increase significantly for all the treatments. The patterns of the observed variations were in accordance with those reported by Giacalone and Chiabrando (2013) for strawberries and by D'Aquino et al. (2010) for filmwrapped pomegranates $(10,5)$.

At the end of the storage time, air-stored 'YousefKhani' arils showed the lowest loss of phenolic content (19.22\%). It is worth noting that there was no significant difference between the $\mathrm{C}$ and $\mathrm{B}$ treatments. On the other hand, the TPC of cv. Malas-e-Saveh arils packed with normal atmosphere (with a $0.69 \%$ increase) and those packed under the B treatment (with a $2.12 \%$ increase) was not statistically different in comparison to their initial level. Moreover, no significant difference was observed between the A (with a 9.82\% increase in TPC) and B treatments. 
Sedighe Tavasoli Talarposhti, et al: Effect of MAP on the Fruit properties of Two Pomegranate Cultivars
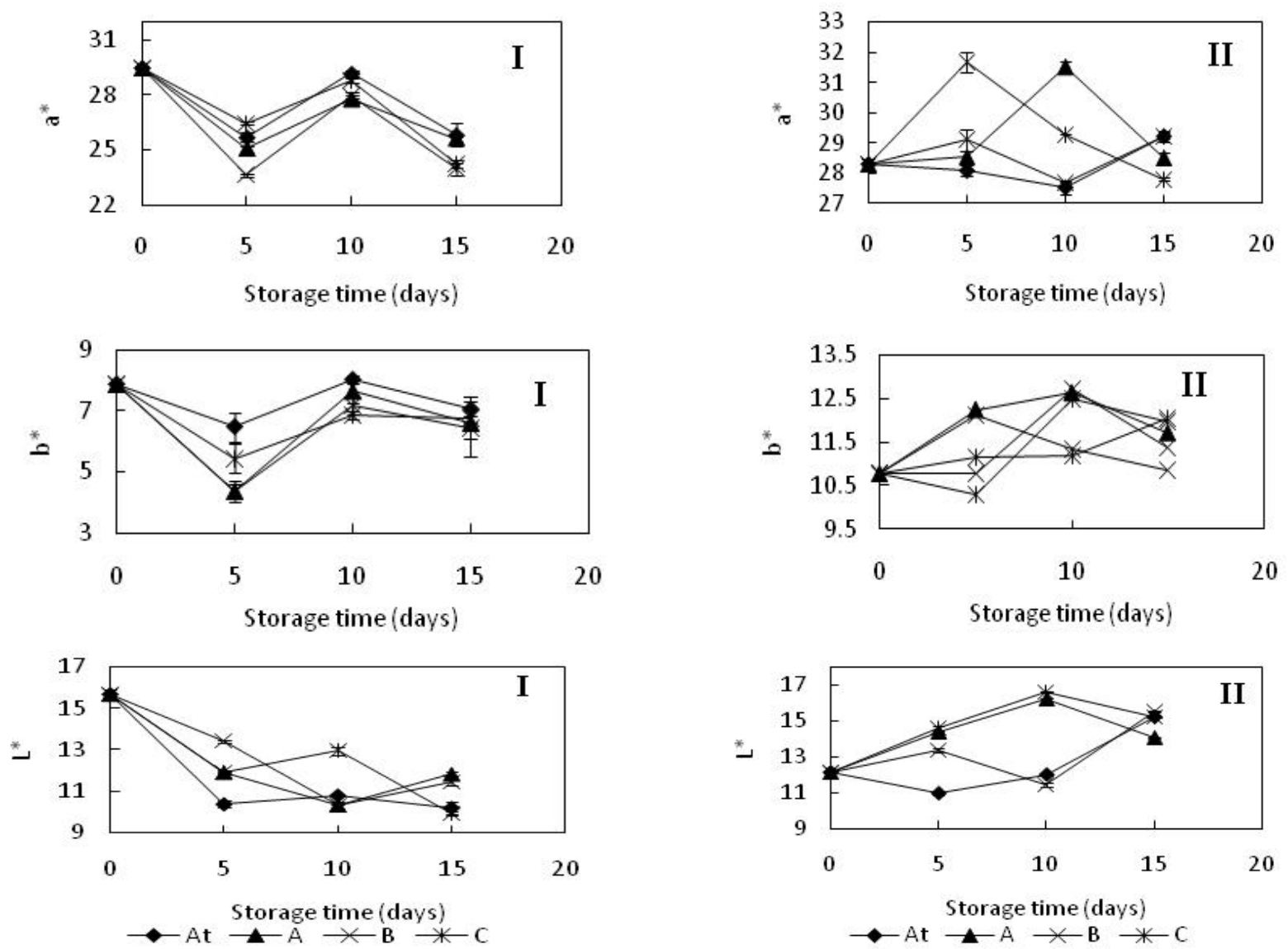

Figure 2. Evaluation of $\mathrm{a}^{*}, \mathrm{~b}^{*}$ and $\mathrm{L}^{*}$ of pomegranate arils packed under modified atmosphere and control during storage. I: cv. Yousef-Khani, II: cv. Malas-e-Saveh; At: normal air; A: 20:5:75 $\mathrm{O}_{2}: \mathrm{CO}_{2}: \mathrm{N}_{2} ; \mathrm{B}: 15: 10: 75 \mathrm{O}_{2}: \mathrm{CO}_{2}: \mathrm{N}_{2} ;$ and C: 10:15:75 $\mathrm{O}_{2}: \mathrm{CO}_{2}: \mathrm{N}_{2}$.
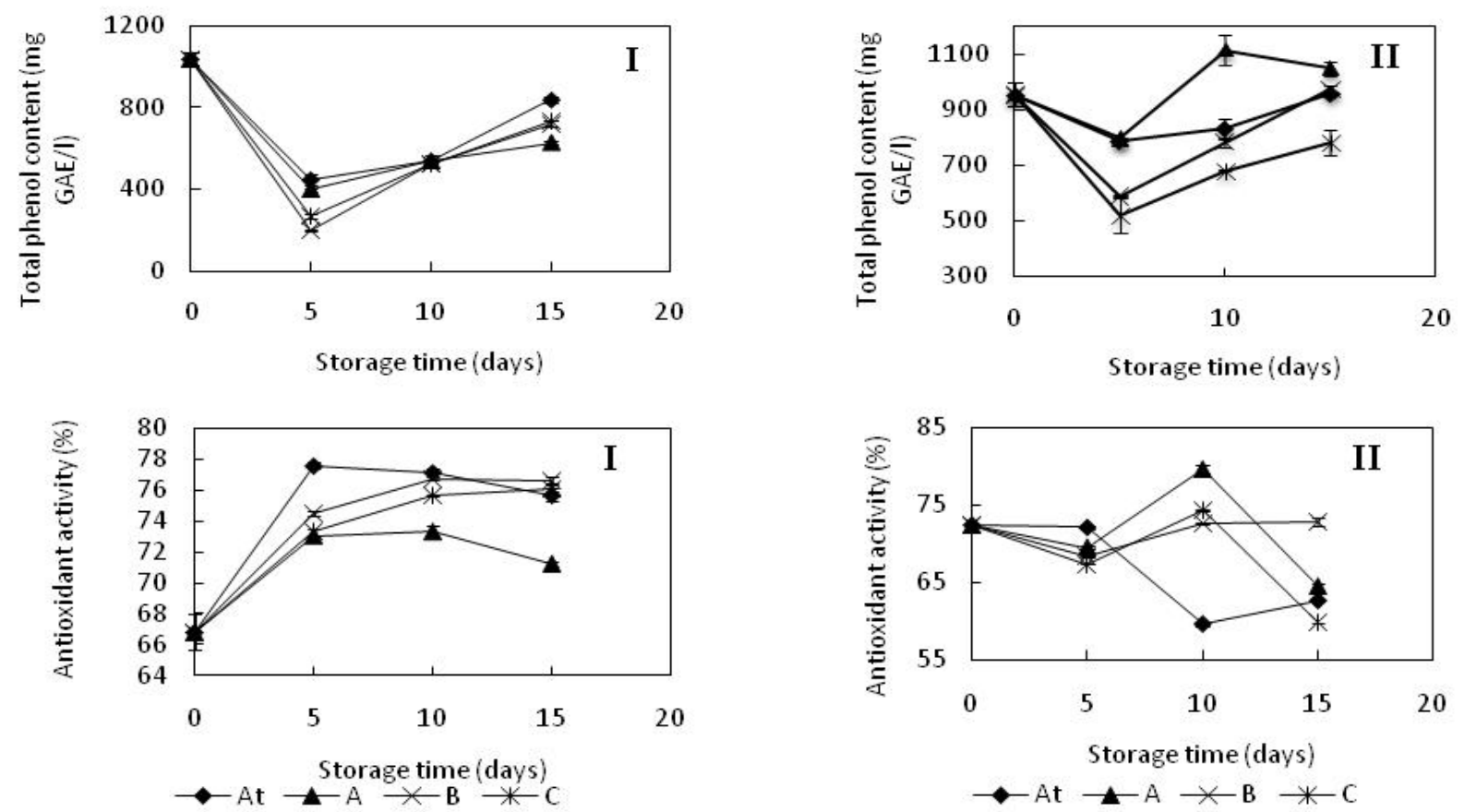

Figure 3. Evaluation of total phenol content and antioxidant activity of pomegranate arils packed under modified atmosphere

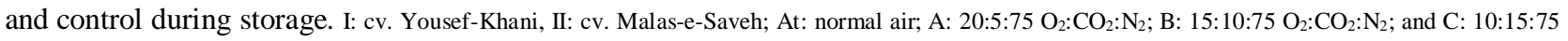
$\mathrm{O}_{2}: \mathrm{CO}_{2}: \mathrm{N}_{2}$ 
Measurement of anthocyanin content: The predominant anthocyanins found in the pomegranate aril juices were as follows: delphinidin 3, 5diglucoside (Dp35) and 3-glucoside (Dp3), cyanidin 3, 5-diglucoside (Cy35), 3-glucoside (Cy3), pelargonidin 3, 5-diglucoside (Pg35), and 3-glucoside (Pg3). The profile of the identified anthocyanins was in accordance with the previous studies $(1,4)$. The initial total anthocyanin content for Yousef-Khani and Malas-e-Saveh cultivars was 686.64 and 563.74 (mg $\left.\mathrm{L}^{-1}\right)$, respectively. The results further indicated a decrease in the anthocyanin content of both cultivars in all the treatments except for air-stored 'Malas-eSaveh' arils and those stored in $15 \% \mathrm{O}_{2}$ plus $10 \%$ $\mathrm{CO}_{2}$ (Figures 4 and 5). At the end of the storage time, there was $13.62 \%$ increase in the total anthocyanin content of 'Malas-e-Saveh' under normal atmosphere; this increase was about $9.25 \%$ under the B treatment $\left(15 \% \mathrm{O}_{2}+10 \% \mathrm{CO}_{2}\right)$. On the other hand, in the case of 'Yousef-Khani' arils, the least decrease in total anthocyanin content was $12.83 \%$ for control; this decrease ranged from 18.68 to $29.71 \%$ for the other treatments.

Table 1 also shows the individual anthocyanin contents of two studied cultivars. According to the data, cyanidin 3, 5-diglucoside was found to be the most representative anthocyanin in both cultivars. Anthocyanins responsible for pigmentation of pomegranate arils were also quantified during the storage time (Figures 4 and 5). The results indicated that neither the different atmospheres nor even the storage time affected the anthocyanins profile. Moreover, during the storage time, cyanidin 3, 5diglucoside had the highest and pelargonidin 3glucoside had the lowest concentration.

After 15 days of storage, the delphinidin, pelargonidin, cyanidin glucoside contents of Malas-eSaveh cultivars stored under normal atmosphere increased by $13-24 \%$, and under the B treatment, this increase amounted to $6-55 \%$. However, there was
$16.43 \%$ decrease in delphinidin-diglucoside value under normal atmosphere and about $28 \%$ under the B treatment. The arils stored under the $\mathrm{A}$ and $\mathrm{C}$ atmospheres showed significant decreases in all anthocyanins, except for delphinidin 3-glucoside under the A atmosphere. Previous studies have indicated that delphinidin glycoside derivatives are among the best substrates for enzymatic oxidation (7), and that the decrease in these anthocyanins was more significant as compared to other anthocyanins. In addition, it has been reported that delphinidin diglucoside derivatives are more stable than monoglucoside ones (4).

The HPLC analysis of Yousef-Khani cultivar showed different results. Mono/di glucoside derivatives of delphinidin and cyanidin decreased under all atmospheres in comparison to the initial values. The lowest loss (12.59-14.31\%) for delphinidin derivatives was recorded under atmospheres B and C. On the other hand, the lowest decrease $(6-23.09 \%)$ in cyanidin derivatives was recorded for the arils packaged under normal atmosphere. Nevertheless, pelargonidin 3, 5diglucoside content increased considerably.

Evaluation of changes in antioxidant activity: The antioxidant activity of fresh-cut 'Yousef-Khani' and 'Malas-e-Saveh' arils was $66.83 \pm 1.16 \%$ and $72.40 \pm 0.10 \%$, respectively. As the data indicate (Figure 3), the antioxidant activity of 'Yousef-Khani' arils increased during the storage time, with the highest recorded value $(12.82 \%)$ was for the arils stored in $15 \% \mathrm{O}_{2}$ plus $10 \% \mathrm{CO}_{2}$. However, the arils stored under the $\mathrm{B}$ and $\mathrm{C}$ treatments did not show a significantly higher antioxidant activity than the control arils. By contrast, the antioxidant activity of 'Malas-e-Saveh' arils decreased. At the end of the storage time, the only treatment that did not result in a significant change in the antioxidant activity of 'Malas-e-Saveh' arils was treatment B.

Table 1. Anthocyanin composition for fresh-cut Malas-e-Saveh and Yousef-Khani cultivars $\left(\mathrm{mg} \mathrm{L}^{-1}\right)^{*}$

\begin{tabular}{lccccccc}
\hline Cultivar & Dp3G & Dp35dG & Pg3G & Pg35dG & Cy3G & Cy35dG & Total \\
\hline Yousef-Khani & 99.65 & 106.33 & 5.66 & 11.99 & 125.72 & 337.27 & 686.64 \\
Malas-e-Saveh & 49.19 & 83.96 & 4.69 & 13.75 & 75.40 & 336.72 & 563.74 \\
\hline
\end{tabular}

"Dp3G: delphinidin-3-glucoside; Dp35dG: delphinidin-3, 5-diglucoside; Pg3G: pelargonidin-3-glucoside; Pg35dG: pelargonidin-3, 5-diglucoside; Cy3G: cyanidin-3-glucoside; and Cy35dG: cyanidin-3, 5-diglucoside. 

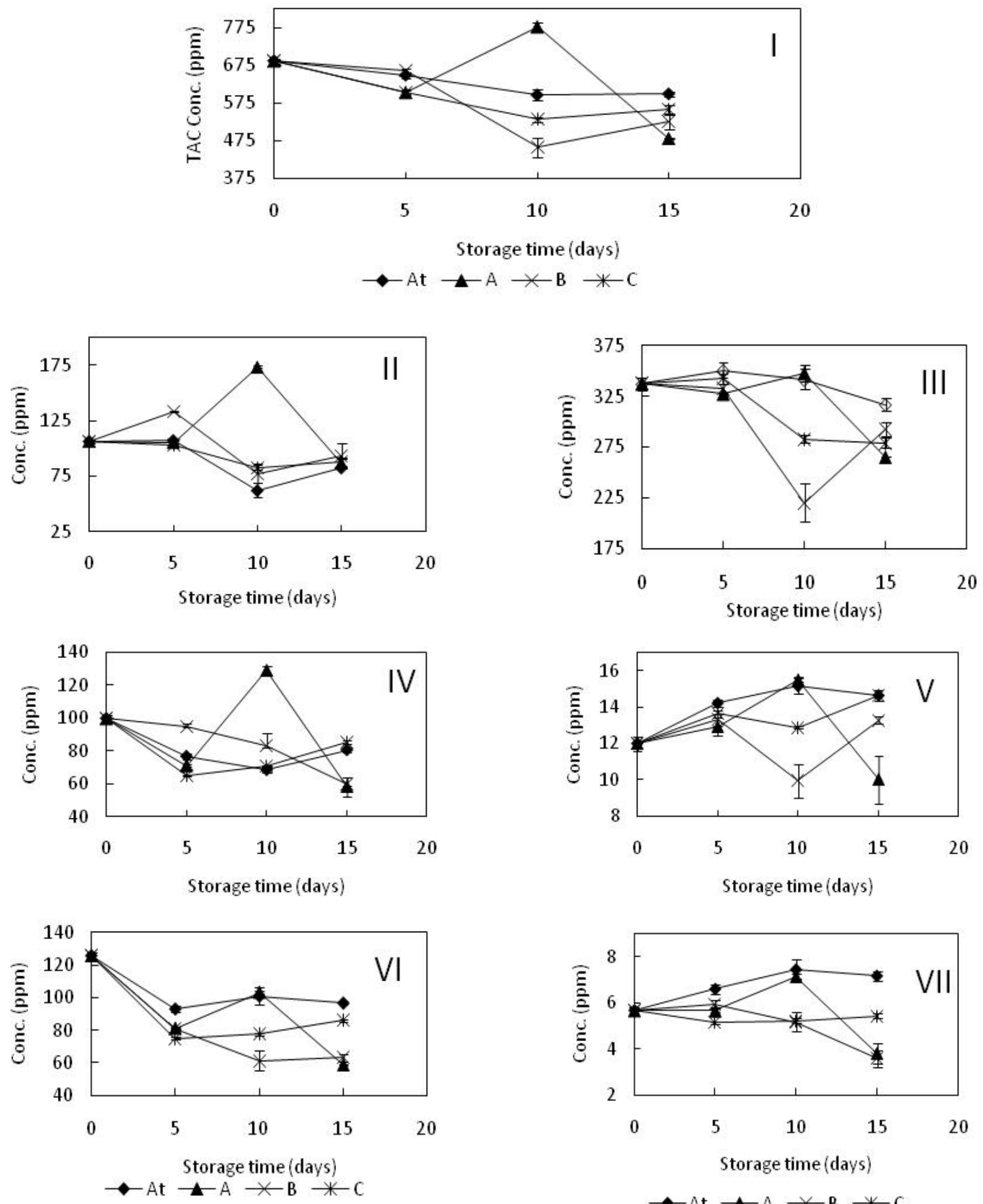

$\bullet$ At $\rightarrow \mathrm{A} \rightarrow \mathrm{B} \rightarrow \mathrm{C}$

Figure 4. Evaluation of total anthocyanin content of pomegranate arils of Yousef-Khani cultivar packed under modified atmosphere and control (I) and individual anthocyanins content during storage. (II: delphinidin-3, 5-diglucoside, III: cyanidin-3, 5diglucoside, IV: delphinidin-3-glucoside, V: pelargonidin-3, 5-diglucoside, VI: cyanidin-3-glucoside and VII: pelargonidin-3-glucoside); At: normal air; A: 20:5:75 $\mathrm{O}_{2}: \mathrm{CO}_{2}: \mathrm{N}_{2} ; \mathrm{B}: 15: 10: 75 \mathrm{O}_{2}: \mathrm{CO}_{2}: \mathrm{N}_{2} ;$ and C: $10: 15: 75 \mathrm{O}_{2}: \mathrm{CO}_{2}: \mathrm{N}_{2}$. 
Sedighe Tavasoli Talarposhti, et al: Effect of MAP on the Fruit properties of Two Pomegranate Cultivars
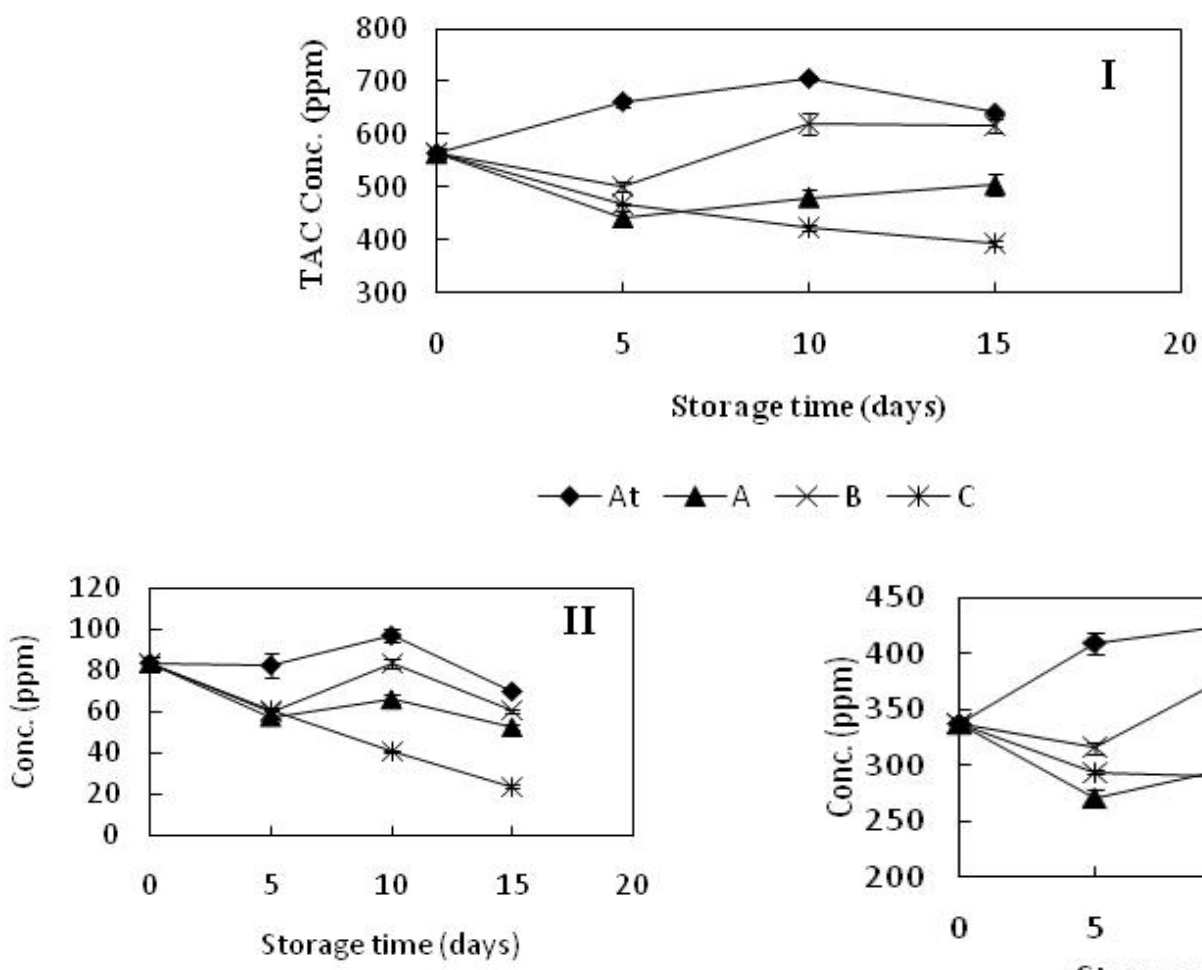

$\rightarrow \mathrm{A} \rightarrow \mathrm{B} \rightarrow \mathrm{C}$
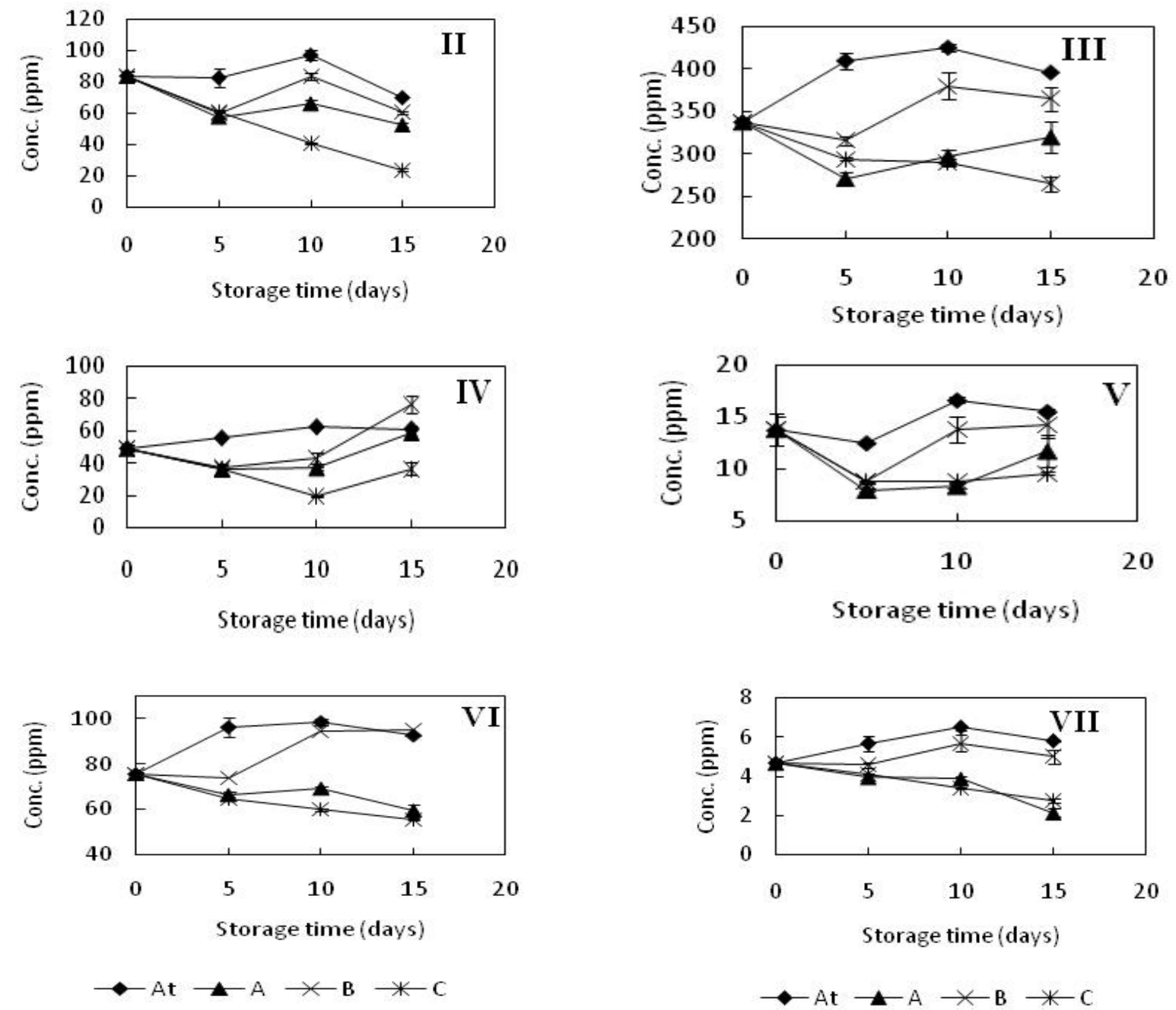

Figure 5. Evaluation of total anthocyanin content of pomegranate arils of Malas-e-Saveh cultivar packed under modified atmosphere and control (I) and individual anthocyanins content during storage (II: delphinidin-3, 5-diglucoside, III: cyanidin-3, 5diglucoside, IV: delphinidin-3-glucoside, V: pelargonidin-3, 5-diglucoside, VI: cyanidin-3-glucoside and VII: pelargonidin-3-glucoside); At: normal air; A: 20:5:75 $\mathrm{O}_{2}: \mathrm{CO}_{2}: \mathrm{N}_{2} ; \mathrm{B}: 15: 10: 75 \mathrm{O}_{2}: \mathrm{CO}_{2}: \mathrm{N}_{2}$; and C: 10:15:75 $\mathrm{O}_{2}: \mathrm{CO}_{2}: \mathrm{N}_{2}$. 
Changes in microbial property: As mentioned before, the standard total plate count method was used to enumerate total microbial populations. The initial number of microorganisms in the pomegranate juice for 'Malas-e-Saveh' and 'Yousef-Khani' arils was 2.26 and $2.67 \log$ colony forming units (cfu) $\mathrm{mL}^{-1}$, respectively. The arils treated with $15 \% \mathrm{O}_{2}+10 \%$ $\mathrm{CO}_{2}$ and also those stored in $10 \% \mathrm{O}_{2}$ plus $15 \% \mathrm{CO}_{2}$ showed the lowest count both during the storage period and at the end of the storage time as compared to those treated with higher $\mathrm{O}_{2}$ concentrations (Figure $6)$. It is worth noting that no significant microbial growth $\left(<10 \mathrm{cfu} \mathrm{mL}^{-1}\right)$ was observed for the arils treated with the above gas compositions from the $5^{\text {th }}$ day to the end of the storage period. Moreover, the differences in the microbial load for these two conditions were not statistically significant. However, the air-stored arils and those treated with $20 \% \mathrm{O}_{2}$ plus $5 \% \mathrm{CO}_{2}$ showed higher counts.
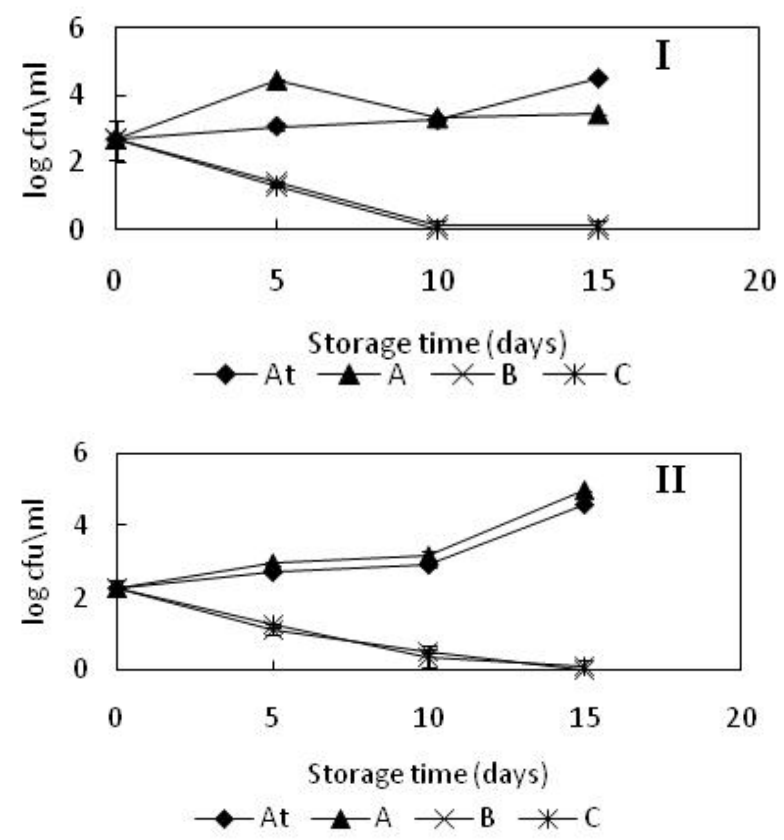

Figure 6. Evaluation of microbial load of pomegranate arils packed under modified atmosphere and control during storage. I: cv. Yousef-Khani, II: cv. Malas-e-Saveh; At: normal air; A: 20:5:75 $\mathrm{O}_{2}: \mathrm{CO}_{2}: \mathrm{N}_{2} ;$ B: 15:10:75 $\mathrm{O}_{2}: \mathrm{CO}_{2}: \mathrm{N}_{2}$; and C: 10:15:75 $\mathrm{O}_{2}: \mathrm{CO}_{2}: \mathrm{N}_{2}$.

\section{Disc ussion}

Some previous studies $(7,11)$ have demonstrated that different levels of atmosphere (e.g. high levels of $\mathrm{CO}_{2}$ and low levels of $\mathrm{O}_{2}$ ) in the packages may cause an increase in TA concentration. Moreover, this increase could be explained by other factors such as the amount of water loss of fruit samples as well as microbial growth and activity during the storage time.

The small but insignificant increases in TSS could be attributed to moisture loss or increase in sugar concentrations together with the polysaccharides' breakdown during storage $(10,11)$.

Based on our analysis of the bioactive compounds (see below), the changes in the $a^{*}$ (redness) value could be related to the changes in total anthocyanin content during the storage time. Similar to this study, Holcroft et al. (1998) reported that changes in color characteristics of pomegranate arils were correlated with the increase in anthocyanin concentration. They also observed that the arils stored in normal atmosphere had higher red color intensity than those stored in $\mathrm{CO}_{2}$-enriched environments (19).

The initial decrease in the TPC of pomegranate arils could be attributed to the specific activity of polyphenol oxidase and its role in TPC degradation (4). On the other hand, the Folin-Ciocalteu assay, besides estimating phenolic compounds, measures the levels of other compounds such as ascorbic acid and anthocyanins, which are highly unstable and may decompose both during the storage time and after sampling. Therefore, the decrease in TPC could also be due to the presence of these compounds. It is worth mentioning that total anthocyanins for both cultivars in the first five days decreased in most of the treatments as compared to their initial level at harvest.

The synthesis of phenolic compounds and the responsible enzymes in this process can be modulated by application of atmosphere modification. Moreover, the increase in phenolic content during the storage time could be explained by changes in the enzyme activities of phenylalanine ammonia-lyase (PAL) as expressed in Kader (1985)(20). Kader reported that PAL acts as the key enzyme contributing to the biosynthesis of phenolic compounds. Furthermore, some previous studies $(19,21)$ have demonstrated that, in the presence of higher levels of $\mathrm{O}_{2}$, the activity of PAL in pomegranate arils increases significantly. Based on the obtained results for cv. Malas-e-Saveh arils in the present study, it could be argued that certain levels of $\mathrm{CO}_{2}$ may positively affect the TPC of pomegranate arils. Gil et al. (1998) also reported similar results (22).

The pattern of the observed changes in anthocyanin accumulation for cv. Malas-e-Saveh was in line with that of Holcroft et al. (1998) (19). They reported that the anthocyanin concentration of arils from the fruits stored in air with $10 \mathrm{kPa} \mathrm{CO}$ increased after 4-6 weeks, and the anthocyanin concentration of 
pomegranates stored in $20 \mathrm{kPa} \mathrm{CO}$ for 6 weeks was lower than their initial concentration. Gil et al. (1996), however, found a $12.92 \%$ decrease in total anthocyanin content in pomegranate arils after 7 days of storage at $4{ }^{\circ} \mathrm{C}$ and under an atmospheric composition of $13.5 \% \quad \mathrm{O}_{2}$ plus $7.5 \% \quad \mathrm{CO}_{2}$ (7). Moreover, López-Rubira et al. (2005), with regard to pomegranate arils harvested in October, UV-C treated and stored under different MAP conditions (26.9 $29.2 \mathrm{kPa} \mathrm{CO}_{2}$ and $<1 \mathrm{kPa} \mathrm{O}$ ), reported a $2.46 \%$ increase in total anthocyanin content after 15 days of storage (6). The authors further observed $28.73 \%$ decrease in the total anthocyanin content of arils from the same cultivar harvested in December and after 13 days of storage at $3^{\circ} \mathrm{C}$. Gil et al. (1997) and Holcroft and Kader (1999) reported that air and controlled atmosphere with a certain level of $\mathrm{CO}_{2}$ affected and accumulated anthocyanin levels in strawberries, but these changes were progressively reduced in fruit with elevated $\mathrm{CO}_{2}$ levels $(12,21)$. Gil et al. (1998) suggested that fresh processed 'Lollo Rosso' lettuce decreased by $37.47 \%$ in anthocyanin pigment concentration (cyanidin 3-malonylglucoside) when stored in air and by $69.60 \%$ when MAP-stored in the presence of $8 \% \mathrm{O}_{2}$ and $3 \% \mathrm{CO}_{2}$.

The possible effect of 'atmosphere modification' on the anthocyanins' content of arils can arise from two concurrent factors: the enzymatic oxidation of anthocyanins and their biosynthesis in controlledatmosphere conditions with certain levels of oxygen and carbon dioxide. Based on the findings, it could be concluded that different $\mathrm{CO}_{2}$ and $\mathrm{O}_{2}$ concentrations may have an impact on the degradation of anthocyanins as well as on their synthesis (19). The decrease in anthocyanins' content may occur due to the possible effects of enzymatic oxidation on some phenolic compounds such as Dp 3, 5dG, Dp 3G, Cy 3G, etc. (23). Moreover, Brownmiller et al. (2008) reported that polymerization of anthocyanins may reduce anthocyanins during storage (24). The authors believe that polymeric compounds formed during storage may occur due to the condensation reactions of anthocyanins with other phenolic compounds. On the other hand, in line with the previous findings (19, 20, 21), our findings revealed that one possible reason for increase in anthocyanins such as Pg35dG could be the post-harvest biosynthesis of phenolic compounds, which is, in turn, dependent on the enzyme activity of the biosynthetic pathway, such as PAL in the arils. Furthermore, it appears that the possible effect of atmosphere modification $\left(10-15 \% \mathrm{CO}_{2}\right)$ on the overall concentration of anthocyanins could be dependent on fruit type. The findings of the present study are in accordance with the previously reported results in this regard $(5,19)$.

The increase in total antioxidant activity of cv. Yousef-Khani arils, despite the reduced levels of total phenolics, could be explained by the increased amount of organic acids. Hansawasdi et al. (2006) found similar results in the case of strawberries, indicating the key role organic acids could play in the antioxidant activity (25). However, a reverse trend was observed for cv. Malas-e-Saveh arils, and a decrease in the total antioxidant activity was found. It could be concluded that the correlation between total antioxidant activity, on the one hand, and total phenolic contents and anthocyanins, on the other, depends on the type of the particular cultivar under study. The same result has been found in Usenik et al. (2008) for strawberries (26). Similarly, Ahn et al. (2005) have shown that MAP packaging has a positive effect on maintaining the antioxidative activity of the Chinese cabbage (Brassica Rapa L.) (27).

The microbial analysis may suggest that the use of coating alone, without altering the normal atmosphere, may not prevent subsequent fungal growth. Similar results have been reported for the 'Malas-e-Saveh' arils stored in polyethylene bags and exposed to hot water treatment (28). In other words, it appears that coating causes a highly moisturized atmosphere and provides conditions that make fungal growth easy. On the other hand, higher metabolic activities of the arils treated with normal air or $20 \%$ $\mathrm{O}_{2}$ plus $5 \% \mathrm{CO}_{2}$ may produce more moisture and warmth, and a subsequent microbial growth should be expected. However, higher levels of $\mathrm{CO}_{2}$ (10-15\%) may be effective in inhibiting microbial growth. Martinez-Ferrer et al. (2006), who examined the effect of MAP on minimally processed mango and pineapple fruits, have reported similar findings (13). Bieganska-Marecik (2004) found more or less the same results in the case of apples (29). Both $\mathrm{O}_{2}$ and $\mathrm{CO}_{2}$ have important preventive functions. Based on the findings, it could be argued that oxygen inhibits the growth of anaerobic bacteria; also in the present study, lower concentrations of $\mathrm{O}_{2}$ were found to prevent mold growth as well as the growth of aerobic bacteria. On the other hand, $\mathrm{CO}_{2}$, by reducing enzymatic activities and reactions and also by altering cell membrane permeability (which, in turn, leads to changes in cell $\mathrm{pH}$ and physico-chemical parameters) can inhibit the growth of microorganisms; this 
preventive function increases significantly in temperatures less than $10^{\circ} \mathrm{C}(30)$.

Based on the observed changes in pomegranate juice antioxidant activity, bioactive compounds and microbial growth, packaging of 'Malas-e-Saveh' arils in $15 \% \mathrm{O}_{2}+10 \% \mathrm{CO}_{2}$ (i.e. treatment $\mathrm{B}$ ) is recommended as the desirable gas composition to extend the shelf-life of this cultivar. It is worth noting that, in the case of cv. 'Yousef-Khani', increases in anthocyanin contents and higher antioxidants in the fruits treated with the modified atmospheres B (15\% $\left.\mathrm{O}_{2}+10 \% \mathrm{CO}_{2}\right)$ and $\mathrm{C}\left(10 \% \mathrm{O}_{2}+15 \% \mathrm{CO}_{2}\right)$ were observed at the end of the storage time. Based on the microbial analysis results for 'Yousef-Khani' arils, moderate $\mathrm{CO}_{2}$ concentrations $\left(10 \% \mathrm{CO}_{2}\right)$ can have some additional advantages such as controlling the post-harvest decay. Therefore, to extend the commercial shelf-life, packaging the arils of this cultivar with an atmospheric composition of $15 \% \mathrm{O}_{2}$ $+10 \% \mathrm{CO}_{2}+75 \% \mathrm{~N}_{2}$ or $10 \% \mathrm{O}_{2}+15 \% \mathrm{CO}_{2}+75 \%$ $\mathrm{N}_{2}$ can yield more desirable results compared to air.

\section{Acknowledgement}

The authors extend their thanks to Tarbiat Modares University (TMU) for its financial support.

\section{Financial disclosure}

The authors declare no financial interest.

\section{Funding/Support}

This study was approved by the Tarbiat Modares University.

\section{References}

1. Mousavinejad G, Emam-Djomeh Z, Rezaei K, Khodaparast $\mathrm{MHH}$. Identification and quantification of phenolic compounds and their effects on antioxidant activity in pomegranate juices of eight Iranian cultivars. Food Chem. 2009; 115: $1274-1278$.

2. Viuda-Martos M, Fernández-López J, Pérez-Álvarez JA. Pomegranate and its many functional components as related to human health: A Review. Compr Rev Food Sci F. 2010; 9: $635-654$.

3. Akhavan H, Barzegar M, Weidlich H, Zimmermann BF. Phenolic Compounds and Antioxidant Activity of Juices from Ten Iranian Pomegranate Cultivars Depend on Extraction. Journal of Chemistry. 2015; $1-7$.

4. Alighourchi H, Barzegar M, Abbasi S. Anthocyanins characterization of 15 Iranian pomegranate (Punica granatum L.) varieties and their variation after cold storage and pasteurization. Eur Food Res Technol. 2008; 227: $881-887$.

5. D’Aquino S, Palma A, Schirra M, Continella A, Tribulato E, Malfa SL. Influence of film wrapping and fludioxonil application on quality of pomegranate fruit. Postharvest Biol Technol. 2010; 55: 121 - 128.
6. López-Rubira V, Conesa A, Allende A, Artés F. Shelf life and overall quality of minimally processed pomegranate arils modified atmosphere packaged and treated with UVC. Postharvest Biol Technol. 2005; 37: $174-185$.

7. Gil MI, Artés F, Tomás-Barberán FA. Minimal processing and modified atmosphere packaging effects on pigmentation of pomegranate seeds. J Food Sci. 1996; 61: $161-164$.

8. Ohlsson T. Minimal processing-preservation methods of the future: an overview. Trends Food Sci Technol. 1994; 5: $341-344$.

9. Church N. Developments in modified-atmosphere packaging and related technologies. Trends Food Sci Technol. 1994; 5: 345 - 352 .

10. Giacalone G, Chiabrando V. Modified atmosphere packaging of sweet cherries with biodegradable films. Int Food Res J. 2013; 20: 1263 - 1268.

11. Ding P, Diana J. Physico-chemical changes in dabai (Canarium odontophyllum Miq.) fruit during modified atmosphere storage. Int Food Res J. 2013; 20: 3033 3040 .

12. Gil MI, Holcroft DM, Kader AA. Changes in strawberry anthocyanins and other polyphenols in response to carbon dioxide treatments. J Agric Food Chem. 1997; 45: 1662 1667.

13. Martínez-Ferrer M, Harper C, Pérez-Muñoz F, Chaparro M. Modified atmosphere packaging of minimally processed mango and pineapple fruits. Food Sci. 2006; 67: $3365-3371$.

14. Garcia E, Salazar DM, Melgarejo P, Coret A. Determination of the respiration index and of the modified atmosphere inside the packaging of minimally processed products. In: Options Méditerranéennes. Série A, Séminaires Méditerranéens. 2000; p. 247 - 51.

15. Alighourchi H, Barzegar M. Some physicochemical characteristics and degradation kinetic of anthocyanin of reconstituted pomegranate juice during storage. J Food Eng. 2009; 90: 179 - 185.

16. Del Carpio Jiménez C, Serrano Flores C, He J, Tian Q, Schwartz SJ, Giusti MM. Characterization and preliminary bioactivity determination of Berberis boliviana Lechler fruit anthocyanins. Food Chem. 2011; 128: 717 - 724 .

17. Alighourchi H, Barzegar M, Abbasi S, Sahari MA. Effect of sonication on anthocyanins, total phenolic content, and antioxidant capacity of pomegranate juices. Int Food Res J. 2013; 20: 1703 - 1709 .

18 Tezcan F, Gültekin-özgüven M, Diken T, Özcelik B, Erim FB. Antioxidant activity and total phenolic, organic acid and sugar content in commercial pomegranate juices. Food Chem. 2009; 115: 873 - 877.

19. Holcroft DM, Gil MI, Kader AA. Effect of carbon dioxide on anthocyanins, phenylalanine ammonia lyase and glucosyl transferase in the arils of stored 
pomegranates. J Am Soc Hortic Sci. 1998; 123: 136 140.

20. Kader AA. Postharvest biology and technology: an overview. In: Postharvest Technology of Horticultural Crops. Cooperative Extension University of California. 1985 ; p. $50-70$.

21. Holcroft DM, Kader AA. Carbon dioxide-induced changes in colour and anthocyanin synthesis of stored strawberry fruit. Hort. Sci. 1999; 34: 1244 - 1248.

22. Gil MI, Castaner M, Ferreres F, Artés F, Tomás-Barberán A. Modified-atmosphere packaging of minimally processed Lollo Rosso (Lactuca sativa). Z Lebensm Unters Forsch. 1998; 206: 350 - 354.

23. Artés F, Tudela JA, Villaescusa R. Thermal postharvest treatments for improving pomegranate quality and shelf life. Postharvest Biol Technol. 2000; 18: 245 - 251.

24. Brownmiller C, Howard LR, Prior RL. Processing and storage effects on monomeric anthocyanins, percent polymeric colour, and antioxidant capacity of processed blueberry products. J Food Sci. 2008; 73: 72 - 79.

25. Hansawasdi C, Rithiudom S, Chaiprasart P. Quality and antioxidant activity changes during low-temperature storage of strawberry fruits. Acta Hortic. 2006; 708: 301 -306 .

26. Usenik V, Fabcic J, Stampar F. Sugars, organic acids, phenolic composition and antioxidant activity of sweet cherry. Food Chem. 2008; 107: 185-192.

27. Ahn HJ, Kim JH, Kim JK, Kim DH, Yook HS, Byun MW. Combined effects of irradiation and modified atmosphere packaging on minimally processed Chinese cabbage (Brassica rapa L.). Food Chem. 2005; 89: 589 597.

28. Talaie A, Askari-Sarcheshmeh MA, Bahadoran F, Sherafatian D. The effects of hot water treatment and in polyethylene bag packaging on the storage life and quality of pomegranate (Cv: Malas- Saveh). J Agrc Sci. 2004; 35: $369-377$.

29. Biegańska-Marecik R, Czapski J, Czaczyk K. The effect of modified atmosphere packaging on the quality of minimally processed apples. EJPAU. 2004; 7(2).

30. Dixon NM, Kell DB. The inhibition by $\mathrm{CO} 2$ of the growth and metabolism of micro-organisms. J Appl Bacteriol. 2008; 67: $109-136$. 